\title{
VARIANTS IN MITOCHONDRIAL tRNA GENE MAY NOT BE ASSOCIATED WITH THYROID CARCINOMA
}

\author{
Lv $\mathrm{F}^{1, \mathrm{a}}$, Qian $\mathrm{G}^{2, \mathrm{a}}$, You $\mathrm{W}^{1, \mathrm{a}}$, Lin $\mathrm{H}^{3}$, Wang $\mathrm{XF}^{3}$, Qiu GS ${ }^{2}$,
} Jiang $\mathrm{YS}^{2}$, Pang $\mathrm{LX}^{3}$, Kang $\mathrm{YM}^{4}$, Jia $\mathrm{BF}^{4}, \mathrm{Xu} \mathrm{JZ}^{5, *}$, Yu Y ${ }^{1, *}$

\begin{abstract}
*Corresponding Authors: Dr. Jinzhong Xu, Department of Clinical Pharmacy, the Affiliated Wenling Hospital of Wenzhou Medial University, Taiping Nan Road 190, Wenling 317500, People's Republic of China. Tel./Fax: +86-(0)576-8620-6288. E-mail: xujzwl@163.com and Dr. Yang Yu, Department of Breast Surgery, Henan Provincial People's Hospital, Weiwu Road 7, Zhengzhou 450003, People's Republic of China. Tel./Fax: +86-(0)3716558-0014. E-mail: 510790135@qq.com
\end{abstract}

\begin{abstract}
Thyroid cancer is a very common form of endocrine system malignancy. To date, the molecular mechanism underlying thyroid cancer remains poorly understood. Studies of oncocytic tumors have led to a hypothesis which proposes that defects in oxidative phosphorylation (OX- PHOS) may result in a compensatory increase in mitochondrial replication and gene expression. As a result, mitochondrial DNA (mtDNA) mutation analysis has become a useful tool to explore the molecular basis of this disease. Among these mutations, mitochondrial transfer RNAs (mttRNAs) are the hot spots for pathogenic mutations associated with thyroid cancer. However, due to its high mutation rate, the role of mt-tRNA variants in thyroid cancer is still controversial. To address this problem, in this study, we reassessed seven reported mt-tRNA variants: tRNA ${ }^{\text {Asp }}$ G7521A, tRNA ${ }^{\text {Arg }}$ T10411C and T10463C, tRNA ${ }^{\text {Leu(CUN) }}$ A12308G, tRNA ${ }^{\text {Ile }}$ G4292C and C4312T, and tRNA ${ }^{\text {Ala }}$ T5655C, in clinical mani-
\end{abstract}

\footnotetext{
${ }^{a}$ F. Lv, G. Qian and W. You contributed equally to this study.

${ }^{1}$ Department of Breast Surgery, Henan Provincial People's Hospital, Zhengzhou, People's Republic of China

2 Department of Endocrinology, Ningbo Fourth Hospital, Xiangshan, People's Republic of China

${ }^{3}$ College of Laboratory Medicine and Life Sciences, Wenzhou Medical University, Wenzhou, People's Republic of China

${ }^{4}$ School of Basic Medical Sciences, Inner Mongolia Medical University, Hohhot, People's Republic of China

${ }^{5}$ Department of Clinical Pharmacy, the Affiliated Wenling Hospital of Wenzhou Medial University, Wenling, People's Republic of China
}

festations of thyroid cancer. We first performed the phylogenetic conservation analysis for these variants; moreover, we used a bioinformatic tool to compare the minimum free energy $(\mathrm{G})$ of mt-tRNA with and without mutations. Most strikingly, none of these variants caused the significant change of the $G$ between the wild-type and the mutant form, suggesting that they may not play an important roles in thyroid cancer. In addition, we screened the frequency of the "pathogenic" A12308G alternation in 300 patients with thyroid cancer and 200 healthy controls. We found that there were five patients and three control subjects carrying this variant. It seemed that the A12308G variant may be a common polymorphism in the human population. Taken together, our study indicated that variants in mt-tRNA genes may not play active roles in patients with thyroid cancer.

Keywords: Association; Mitochondrial DNA (mtDNA); Thyroid cancer; Mitochondrial transfer RNA (mt-tRNA); Variants

\section{INTRODUCTION}

With an incidence of $2.0 \%$, thyroid carcinoma is the most common form of endocrine system malignancy $[1,2]$. Thyroid carcinomas are histologically classified as papillary thyroid carcinoma (PTC), follicular thyroid carcinoma (FTC), anaplastic thyroid carcinoma (ATC) and medullary thyroid carcinoma (MTC), accounting for approximately 80.0, 15.0, 2.0 and $4.0 \%$ of all thyroid malignancies, respectively [3]. Decreased survival in patients with oncocytic 
carcinomas may be due to reduced competence in iodine uptake by the tumor cells, resulting in poor response to radioiodine treatment. However, to date, the molecular mechanism underlying this disease remain largely unknown.

Since Warburg proposed that cancer originated from a non neoplastic cell that adopted anaerobic metabolism as a means of survival after injury to its respiratory system [4], changes in the number, shape, and function of mitochondria have been reported in various cancers [5]. The mitochondrial genome is a closed double-stranded circular molecule consisting of 16,569 bp coding for 37 genes, including 13 polypeptides, 22 tRNAs and two rRNAs necessary for function of the respiratory chain [6]. Due to the lack of histone protection and a poor repair system, mtDNA is thought to be more susceptible than nuclear DNA to mutagen-induced damage [7]. Of these, mt-tRNA is the hot-spot for mutations in cancers as it is preferentially damaged by many carcinogens [8]. However, some of these mutations are single nucleotide polymorphisms (SNPs) and may not cause mitochondrial dysfunction, such as the mttRNA $^{\text {Phe }}$ C628T variant in deafness expression [9]. Distinguishing the SNPs and mutations is important, because failure to do so will inevitably lead to poor diagnosis and genetic advice.

In this study, we reassess seven reported mttRNA variants: tRNA ${ }^{\text {Asp }}$ G7521A, tRNA ${ }^{\text {Arg }}$ T10411C and $\mathrm{T} 10463 \mathrm{C}$, tRNA ${ }^{\mathrm{Leu}(\mathrm{CUN})} \mathrm{A} 12308 \mathrm{G}$, tRNA ${ }^{\text {Ile }}$ G4292C and C4312T, tRNA ${ }^{\text {Ala }}$ T5655C, in clinical manifestation of thyroid cancer. First, we carried out database searches for the allele frequencies of these variants, and then the genotype to phenotype association of these variants. Moreover, we performed the phylogenetic conservation analysis of these variants. We further utilized the bioinformatic tool to predict the $\Delta \mathrm{G}$ of mt-tRNAs with and without these variants. To determine the frequency of $\mathrm{A} 12308 \mathrm{G}$ variant, we screened this variant in 300 patients with thyroid cancer and 200 controls.

\section{MATERIALS AND METHODS}

Database Searches. We systematically searched for unrestricted language articles included in PubMed, Embase, Google Scholar, Cochrane Library, China National Knowledge Infrastructure (CNKI), Chinese VIP, and Chinese Wan-fang databases from inception to December 2015. The literature search in PubMed was carried out using the following keywords "mitochondrial tRNA mutations, thyroid carcinoma," or "mt-tRNA mutations, thyroid cancer," or "mt-tRNA variants, thyroid cancer." We excluded studies if the crucial data were not reported in original papers, or if they had a very high probability of inaccurate reporting.

Data Extraction. Data were independently extracted by two authors (F. Lv and G. Qian), and checked by another author (W. You). Any disagreement was resolved by discussions until consensus was reached.

Determining the Conservation Index of These mt-tRNA Variants. With the purpose of understanding the possible role of mt-tRNA variants in thyroid cancer, we performed a phylogentic approach to see the conservation index (CI) of each mt-tRNA variant. In brief, the mtDNA sequences of 15 vertebrates were used in the inter-specific analyses; these species included Mus musculus, Gorilla gorilla, Hylobates lar, Lemur catta, Pan paniscus, Homo sapiens, Thylamys elegans, Procavia capensis, Cavia porcellus, Orycteropus afer, Bos taurus, Sus scrofa, Felis catus, Platanista minor and Herpestes javanicus. The CI was then calculated by comparing the human nucleotide (nt) variants with 14 other vertebrates. Notably, the $\mathrm{CI}$ of $>70.0 \%$ was regarded to have a functional potential [10].

Calculation of the $\triangle \mathbf{G}$ of mt-tRNA With and Without These Variants. To see whether these variants affected the $\Delta \mathrm{G}$ of mt-tRNAs, we used the RNA Fold Web server program to measure the $\Delta \mathrm{G}$ of each mt-tRNA with and without these variants (http://rna. tbi.univie.ac.at/cgi-bin/ RNAfold. cgi) [11].

Screening for the Frequency of the A12308G Variant in Thyroid Carcinoma. We then performed the polymerase chain reaction (PCR)-Sanger sequence to detect the occurrence of the "well-known" A12308G variant in patients with thyroid cancer. Briefly, a total of 300 blood samples of thyroid cancer (150 male and 150 females; average age 52 years) and 200 healthy subjects ( 100 males and 100 females; average age 49 years) were recruited from the Henan Provincial People's Hospital, Zhengzhou, People's Republic of China (PRC). Informed consent and clinical evaluation were obtained from all these subjects. The study protocol was approved by the Ethics Committee of Henan Provincial People's Hospital. 
We first extracted the genomic DNA from each sample, then we used the PCR to amplify the gene using the following primer sequences: forward (5'-TGC TAG TAA CCA CGT TCT CC-3'); reverse (5'-TTT GTT AGG GTT AAC GAG GG-3'). The PCR product was subsequently examined for specificity using $1.5 \%$ agarose gel electrophoresis. Double-stranded automated sequencing was performed using an $\mathrm{ABI}$ PRISM $^{\text {TM }} 3700$ sequencing machine (Applied Biosystems Inc., Foster City, CA, USA). The sequence was then compared with the human mitochondrial reference sequence (NC_012920) [6].

\section{RESULTS}

Study Characteristics. As a result, three studies were identified using the keywords mentioned in the previous section. After a full-text review, we noticed that one of them concerned the somatic $\mathrm{mtD}$ NA mutations in PTC [12]. Another article that met our inclusion criteria was about the association of mtDNA transversion mutations with familiar MTC [13], whereas another article talked mainly about the mtDNA mutations causing defective OXPHOS in thyroid carcinoma [14].

Selecting Thyroid Carcinoma Associated mttRNA Variants. We further screened the mt-tRNA variants that had been reported with thyroid cancer. Consequently, a total of seven mt-tRNA variants were described: tRNA ${ }^{\text {Asp }}$ G7521A, tRNA ${ }^{\text {Arg }}$ T10411C and T10463C, tRNA ${ }^{\text {Leu(CUN) }}$ A12308G, tRNA ${ }^{\text {Ile }}$ G4292C and $\mathrm{C} 4312 \mathrm{~T}$, and tRNA ${ }^{\text {Ala }} \mathrm{T} 5655 \mathrm{C}$. The molecular characterization of these mt-tRNA variants are listed in Table 1 and Figure 1.

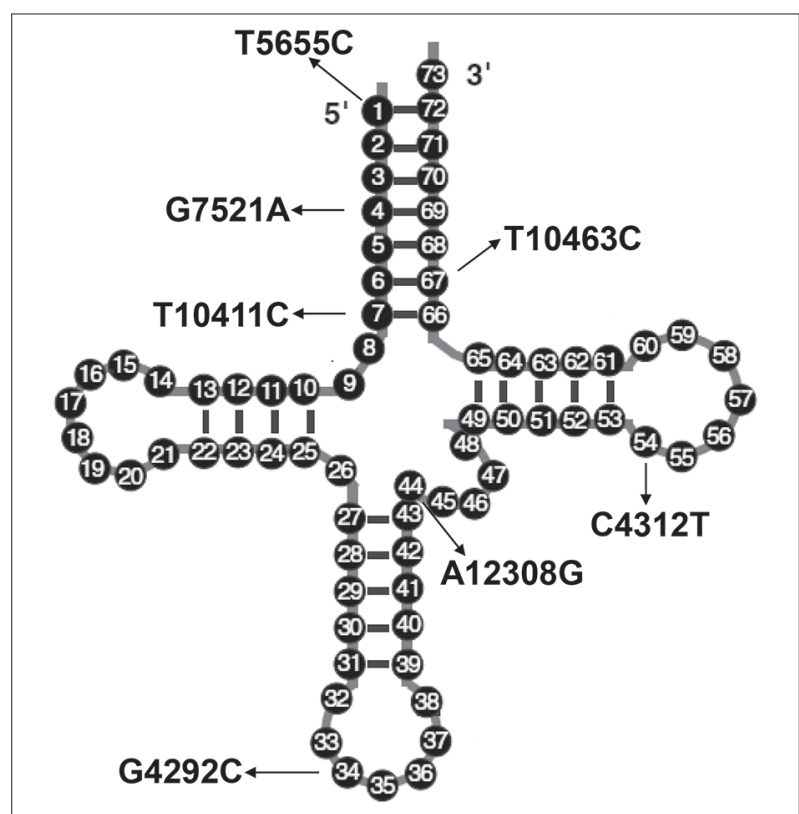

Figure 1. Mitochondrial tRNA mutations with standard nt numbering, arrows indicate the locations of mt-tRNA variants.

Conservation Assessment of These Variants. Assessing pathogenicity of a nt substitution in a mttRNA gene involved evaluation of the evolutionary conservation of the base involved. For this purpose, we analyzed the CIs of these mt-tRNA variants. Briefly, we chose 15 animals for inter-species analysis, these species included Mus musculus, Gorilla gorilla, Hylobates lar, Lemur catta, Pan paniscus, Homo sapiens, Thylamys elegans, Procavia capensis, Cavia porcellus, Orycteropus afer, Bos taurus, Sus scrofa, Felis catus, Platanista minor and Herpestes javanicus. We used the sequences derived from mammalian mitochondrial tRNA genes (http://mamit-trna.u-

Table 1. Molecular characterization of mt-tRNA variants for thyroid cancer.

\begin{tabular}{|l|c|c|c|c|c|c|c|}
\hline \multirow{2}{*}{ tRNA } & \multirow{2}{*}{ tRNA $^{\text {Asp }}$} & \multicolumn{2}{|c|}{ tRNA $^{\text {Arg }}$} & tRNA & \multicolumn{2}{c|}{ tRNA $^{\text {Ile }}$} & tRNA $^{\text {Ala }}$ \\
\hline Nt changes & G7521A & T10411C & T10463C & A12308G & G4292C & C4312T & T5655C \\
\hline $\begin{array}{l}\text { Number of } \\
\text { nts in tRNA }\end{array}$ & 4 & 7 & 67 & 44 & 34 & 54 & 1 \\
\hline Position & acceptor arm & acceptor arm & acceptor arm & variable loop & anticodon arm & T arm & acceptor arm \\
\hline $\begin{array}{l}\text { Homoplasmy/ } \\
\text { heteroplasmy }\end{array}$ & homoplasmy & homoplasmy & homoplasmy & homoplasmy & homoplasmy & homoplasmy & homoplasmy \\
\hline CI (\%) & 13.5 & 21.1 & 94.2 & 93.3 & 10.0 & 19.0 & 71.2 \\
\hline $\begin{array}{l}\text { G (wild type) } \\
\text { (kcal/mol) }\end{array}$ & -10.59 & -10.58 & -10.86 & -16.01 & -8.98 & -8.98 & -18.47 \\
\hline $\begin{array}{l}\text { G (mutant) } \\
\text { (kcal/mol) }\end{array}$ & -11.11 & -10.13 & -10.13 & -15.88 & -9.06 & -9.12 & -18.81 \\
\hline
\end{tabular}

CI: conservation index. 


\begin{tabular}{|c|c|c|c|c|c|c|c|c|c|c|c|c|c|c|c|}
\hline \multirow[t]{2}{*}{ Organism } & \multicolumn{2}{|l|}{$\begin{array}{l}\text { Acc- } \\
\text { stem }\end{array}$} & \multirow{2}{*}{$\begin{array}{l}\text { D-stem } \\
10\end{array}$} & \multirow[t]{2}{*}{ D-1oop } & \multicolumn{2}{|l|}{ D-stem } & \multirow{2}{*}{$\begin{array}{c}\text { Ac- } \\
\text { stem } \\
27\end{array}$} & \multirow{2}{*}{$\begin{array}{l}\text { Anticd- } \\
\text { loop } \\
32\end{array}$} & \multirow{2}{*}{$\begin{array}{l}\text { Ac-stem } \\
39\end{array}$} & $\begin{array}{c}\text { V- } \\
\text { region }\end{array}$ & T-stem & \multirow[t]{2}{*}{ T-loop } & T-stem & \multicolumn{2}{|l|}{$\begin{array}{l}\text { Acc- } \\
\text { stem }\end{array}$} \\
\hline & 1 & 8 & & & 22 & & & & & & 49 & & 61 & 66 & 73 \\
\hline Mus musculus & ACTTTIA & TA & GGAT & AATAGTA & ATCC & $A$ & TTGGT & CTTAGGA & ACCAA & AAAC & CTTGG & TGCAAAT & CCAAA & TAAAAAGT & $A$ \\
\hline Gorilla gorilla & ACTTTTA & $A A$ & GGAT & AACAGCT & ATCC & A & TTGGT & CTTAGGA & CCCAA & AAAT & TTTGG & TGCAACT & CCAAA & TAAAAGT & A \\
\hline Hylobates lar & ACTTTTA & A.A & GGAT & AACAGCT & ATCC & $A$ & TTGGT & CTTAGGA & CCCAA & A.A.AT & TTTGG & TGCAACT & CCAAA & TAAAAAGT & $A$ \\
\hline Lemur catta & ACTTTTA & AA & GGAT & AGAAGTA & ATCC & $A$ & TTGGC & CTTAGGA & GCCAA & AAA & ATTGG & TGCAACT & CCAAA & TAAAAGT & A \\
\hline Pan paniscus & ACTTTTA & $A A$ & GGAT & AACAGCC & ATCC & $\mathrm{G}$ & TTGGT & CTTAGGC & CCCAA & AAAT & TTTGG & TGCAACT & CCAAA & TAAAAGT & A \\
\hline Homo sapiens & ACTTTTA & AA & GGAT & AACAGCT & ATCC & A & TTGGT & CTTAGGC & CCCAA & AAAT & TTTGG & TGCAACT & CCAAA & TAAAAAGT & A \\
\hline Thylarnys elegans & ACTTTTA & A.A & GGAT & AGCAGTA & ATCC & $\mathrm{c}$ & CTGGT & CTTAGGA & ACCAG & TAAC & ITTGG & TGCAACT & CCAAA & TAAAAAGT & $A$ \\
\hline Procavia capensis & ACTTTTA & $A A$ & GGAT & GGGAGAT & ATCC & $\mathrm{G}$ & TTGGT & CTTAGGA & GCCAA & AAA & ATTGG & TGCAACT & CCAAA & TAAAAGT & $A$ \\
\hline Cavia porcellus & ACTTTTA & TA & GGAT & AGAAGTA & ATCC & $A$ & TTGGT & CTTAGGA & ACCAA & AAA & ATTGG & TGCAACT & CCAAA & TAAAAGT & $A$ \\
\hline Orycteropus afer & ACTTTTA & TA & GGAT & AGTAGTA & ATCC & G & TTGGT & CTTAGGA & ACCAA & A.A.A & ATTGG & TGCAACT & CCAAA & TAAAAGT & $A$ \\
\hline Bos taurus & ACTTTTA & $A A$ & GGAT & AGTAGTTT & ATCC & G & TTGGT & CTTAGGA & ACCAA & AAA & ATTGG & TGCAACT & CCAAA & TAAAAGT & $A$ \\
\hline Sus scrofa & ACTTTTA & $A A$ & GGAT & AACAGCT & ATCC & G & TTGGT & CTTAGGA & ACCAA & AAA & ATTGG & TGCAACT & CCAAA & TAAAAGT & $A$ \\
\hline Felis catus & ACTTTTA & TA & GGAT & AGAAGTA & ATCC & $A$ & TTGGT & CTTAGGA & ACCAA & A.AA & ATTGG & TGCAACT & CCAAA & TAAAAGT & $A$ \\
\hline Platanista minor & ACTTTTA & $A A$ & GGAT & A.ATAGTT & ATCC & $A$ & TTGGC & CITAGGA & GCCAA & A.AA & ATTGG & TGCAAGT & CCAAA & TAAAAGT & A \\
\hline Herpestes javanicus & ACTTTTA & TA & GGAT & AGAAGTA & ATCC & G & ITGGT & CTTAGGA & ACCAA & AAA & ATTGG & TGCAACT & CCAAA & IGAAAGT & $A$ \\
\hline
\end{tabular}

Figure 2. Phylogenetic conservation analysis of the A12308G variant (the arrow shows position 44 that corresponds to the A12308G variant).

strasbg.fr/) [15]. The CI was calculated by comparing the corresponding $n t$ at the position of mt-tRNA. We found that the T10463C, A12308G and T5655C variants exhibited the highest level of CI, whereas other mutations lacked high level of CIs. Notably, the CI of the $\mathrm{A} 12308 \mathrm{G}$ mutation is $93.3 \%$ (Figure 2).

Prediction of the $\Delta \mathbf{G}$ of Each mt-tRNA. We used the RNA Fold Web Server (http://rna.tbi.univie. ac.at/cgi-bin/ RNAfold. cgi) to calculate the $\Delta \mathrm{G}$ of each mt-tRNA with and without these mutations, as listed in Table 1. Surprisingly, none of these variants caused significant alternation of $\Delta \mathrm{G}$ between the wildtype and the mutant mt-tRNAs, it seemed that they may not play important roles in thyroid carcinoma.

Mutational Analysis of the tRNA ${ }^{\mathrm{Leu}(\mathrm{CUN})}$ Gene. By comparing the human mitochondrial genome sequence, we found that there were five thyroid cancer patients and three control subjects carrying the homoplasmic A12308G variant, suggesting that it may be very polymorphic in the human population.

\section{DISCUSSION}

Mutations of mtDNA, as well as nuclear genome, are associated with various human diseases and may play important roles in age-related disorders, including cancer and aging [16]. Alterations in OXPHOS in tumor cells were originally believed to play a causative role in malignant growth and tumorigenesis [17]. On the other hand, mutations in the mt-tRNA genes have impact on the secondary and tertiary tRNA structure, and may consequently cause transcriptional and translational defects and mitochondrial respiratory chain dysfunction. More than half of mitochondrial mutations have been located in mt-tRNA genes which are hot-spots for mitochondrial pathogenesis [18]. However, we noticed that a certain amount of mt-tRNA mutations were wrongly classified as a "pathogenic" mutation, such as the C628T variant in deafness expression [9].

In this study, we evaluated seven reported mttRNA variants with thyroid cancer by employing phylogenetic conservation analysis (Table 1 and Figure 1). Of these variants, four (G7521A, T10411C, T10463C and $\mathrm{T} 5655 \mathrm{C}$ ) were located at the acceptor arm, one variant (A12308G) localized at the variable loop, one variant (G4292C) was in the anticodon loop, while the $\mathrm{C} 4312 \mathrm{~T}$ variant was at the $\mathrm{T}$ arm. By systematic review and literature searching, we found that only the A12308G and T5655C variants had been reported to be associated with mitochondrial diseases, while other variants were rare polymorphisms and had not been reported before. Of these, the common variant, $\mathrm{A} 12308 \mathrm{G}$, had been described with a wide range of clinical disorders such as stroke [19], and this variant may increase the risk of developing pigmentary retinal degeneration, short stature, dysphasia-dysarthria and cardiac conduction defects [20]. In addition, the T5655C variant had been reported to increase the penetrance and expressivity of non syndromic deafness associated with the tRNA $^{\text {Ser(UCN) }}$ T7511C mutation [21]. However, as the $\mathrm{T} 5655 \mathrm{C}$ variant caused a slight change of $\Delta \mathrm{G}$ between the wild-type and the mutant tRNA ${ }^{\text {Ala }}$, it indicated that it may have been served as a polymorphism. 
In order to examine structure-function relationships, we used the RNA Fold Web Server (http:// rna.tbi.univie. ac.at/cgi-bin/RNAfold.cgi) program to predict the optimal secondary structures for the mt-tRNAs through free energy minimization [22,23], in which the size of the minimum free energy change indicated the "driving force" behind the reaction, and the more negative the $\Delta \mathrm{G}$ is, the more likely was the process. We found that a wide spectrum of differences in the $\Delta \mathrm{G}$ of the mt-tRNAs. The largest negative differences in $\Delta \mathrm{G}$ (wild-type) $-\Delta \mathrm{G}$ (mutant) $<-3$ were found in all these variants, suggesting that the polymorphic variant was energetically less favorable than the wild-type form.

Previous studies showed a positive association between the known tRNA ${ }^{\text {Leu(CUN) }} \mathrm{A} 12308 \mathrm{G}$ variant and clinical phenotypes. However, Deschauer et al. [24] considered that this mutation may not increase the risk of stroke in patients with the tRNA ${ }^{\text {Leu(UUR) }}$ A3243G mutation. To address this problem, we performed a mutational analysis for the A12308G in thyroid cancer individuals and healthy controls. To our surprise, there were five patients with this variant, as well as three control subjects. This finding was consistent with a recent study by Mohammed et al. [25], and our data suggested that the $\mathrm{A} 12308 \mathrm{G}$ variant was most probably a neutral polymorphism as it was a common event in the human genetic population.

Analysis of the CIs of these variants showed that three of them exhibited high levels of conservation, including the $\mathrm{A} 12308 \mathrm{G}, \mathrm{T} 10463 \mathrm{C}$ and $\mathrm{T} 5655 \mathrm{C}(\mathrm{CI}$ $>70.0 \%$ ). However, we noticed that not all the pathogenic mutations were conserved between different species, such as the Leber's Hereditary Optic Neuropathy (LHON) associated ND4 G11778A mutation [26]. Most importantly, using the cybrid cells carrying these variants, by examining the steady-state level, aminoacylation ability were the "gold standards" to detect the polymorphisms and mutations [27]. Therefore, based on this study, we found that mt-tRNA variants may not be involved in the pathogenesis of thyroid carcinoma.

Declaration of Interest. This study was partly supported by Zhejiang Provincial Natural Science Foundation (LQ13H280002, LY12H15001 and LY12H03001), Henan Basic and Frontier Technology Research Projects (142300410388 and 132300410048), Wenling Foundation of Science and Technology (2011WLCB0109, 2014C311051 and 2015C312055), Project of Medical Technology of Zhejiang Province (2013KYA130, 2015KYB234, 2015KYA 154, 2016KYA166 and 2016KYB275), Public Project of Science and Technology of Wenzhou City (Y20140739 and Y20150094), Ningbo Natural Science Foundation (2015A610234), and Xiangshan Science and Technology Project (2015C6005). The authors report no conflicts of interest. The authors alone are responsible for the content and writing of this article.

\section{REFERENCES}

1. Su JJ, Hui LZ, Xi CJ, Su GQ. Correlation analysis of ultrasonic characteristics, pathological type, and molecular markers of thyroid nodules. Genet Mol Res. 2015; 14(1): 9-20.

2. Jemal A, Siegel R, Xu J, Ward E. Cancer statistics, 2010. CA Cancer J Clin. 2010; 60(5): 277-300.

3. Hundahl SA, Fleming ID, Fremgen AM, Menck HR. A National Cancer Data Base report on 53,856 cases of thyroid carcinoma treated in the U.S., 1985-1995 [see comments]. Cancer. 1998; 83(12): 2638-2648.

4. Warburg O. On the origin of cancer cells. Science. 1956; 123(3191): 309-314.

5. Pedersen PL. Tumor mitochondria and the bioenergetics of cancer cells. Prog Exp Tumor Res. 1978; 22: 190-274.

6. Anderson S, Bankier AT, Barrell BG, de Bruijn $\mathrm{MH}$, Coulson AR, Drouin J, et al. Sequence and organization of the human mitochondrial genome. Nature. 1981; 290(5806): 457-465.

7. Nesheva D. Aspects of ancient mitochondrial DNA analysis in different populations for understanding human evolution. Balkan J Med Genet. 2014; 17(1): 5-14.

8. Backer JM, Weinstein IB. Mitochondrial DNA is a major cellular target for a dihydrodiol-epoxide derivative of benzo[a]pyrene. Science. 1980; 209(4453): 297-299.

9. Zhu Q, Zhou Y, Jin X, Lin X. The role of mitochondrial tRNAPhe C628T variant in deafness expression. Mitochondrial DNA. 2015; 26(1): 2-6. 
10. Ruiz-Pesini E, Wallace DC. Evidence for adaptive selection acting on the tRNA and rRNA genes of human mitochondrial DNA. Hum Mutat. 2006; 27(11): 1072-1081.

11. Gruber AR, Lorenz R, Bernhart SH, Neuböck R, Hofacker IL. The Vienna RNA Website. Nucleic Acids Res. 2008; 36(Web Server issue): W70W74.

12. Yeh JJ, Lunetta KL, van Orsouw NJ, Moore FD Jr, Mutter GL, Vijg J, et al. Somatic mitochondrial DNA (mtDNA) mutations in papillary thyroid carcinomas and differential mtDNA sequence variants in cases with thyroid tumours. Oncogene. 2000; 19(16): 2060-2066.

13. Abu-Amero KK, Alzahrani AS, Zou M, Shi Y. Association of mitochondrial DNA transversion mutations with familial medullary thyroid carcinoma/multiple endocrine neoplasia type 2 syndrome. Oncogene. 2006; 25(5): 677-684.

14. Bonora E, Porcelli AM, Gasparre G, Biondi A, Ghelli A, Carelli V, et al. Defective oxidative phosphorylation in thyroid oncocytic carcinoma is associated with pathogenic mitochondrial DNA mutations affecting complexes I and III. Cancer Res. 2006; 66(12): 6087-6096.

15. Florentz C, Sohm B, Tryoen-Tóth P, Pütz J, Sissler M. Human mitochondrial tRNAs in health and disease. Cell Mol Life Sci. 2003; 60(7): 1356-1375.

16. Sen HM, Silan F, Silan C, Degirmenci Y, Ozisik Kamaran HI. Effects of CYP2C19 and P2Y12 gene poly-morphisms on clinical results of patients using clopidogrel after acute ischemic cerebrovascular disease. Balkan J Med Genet. 2015; 17(2): 37-41.

17. Cavalli LR, Liang BC. Mutagenesis, tomourigenicity, and apoptosis: Are the mitochondria involved? Mutat Res. 1998; 398(1-2): 19-26.

18. Sternberg D, Danan C, Lombès A, Laforêt $P$, Girodon E, Goossens M, et al. Exhaustive scanning approach to screen all the mitochondrial tRNA genes for mutations and its application to the investigation of 35 independent patients with mitochondrial disorders. Hum Mol Genet. 1998; 7(1): 33-42.

19. Pulkes T, Sweeney MG, Hanna MG. Increased risk of stroke in patients with the A12308G polymorphism in mitochondria. Lancet. 2000; 356(9247): 2068-2069.

20. Crimi M, Del Bo R, Galbiati S, Sciacco M, Bordoni A, Bresolin N, et al. Mitochondrial A12308G polymorphism affects clinical features in patients with single mtDNA macrodeletion. Eur J Hum Genet. 2003; 11(11): 896-898.

21. Li X, Fischel-Ghodsian N, Schwartz F, Yan Q, Friedman RA, Guan MX. Biochemical characterization of the mitochondrial tRNASer(UCN) T7511C mutation associated with nonsyndromic deafness. Nucleic Acids Res. 2004; 32(3): 867-877.

22. Mathews DH. RNA secondary structure analysis using RNAstructure. Curr Protoc Bioinformatics. 2014; 46:12.6.1-12.6.25. doi: 10.1002/0471250953.bi1206s46.

23. Mathews DH, Sabina J, Zuker M, Turner DH. Expanded sequence dependence of thermodynamic parameters improves prediction of RNA secondary structure. J Mol Biol. 1999; 288(5): 911-940.

24. Deschauer M, Chinnery PF, Schaefer AM, Turnbull DM, Taylor RW, Zierz S, et al. No association of the mitochondrial DNA A12308G polymorphism with increased risk of stroke in patients with the A3243G mutation. J Neurol Neurosurg Psychiatry. 2004; 75(8): 1204-1205.

25. Mohammed F, Rezaee Khorasany AR, Mosaieby E, Houshmand M. Mitochondrial A12308G alteration in tRNA(Leu(CUN)) in colorectal cancer samples. Diagn Pathol. 2015; 10: 115.

26. Wallace DC, Singh G, Lott MT, Hodge JA, Schurr TG, Lezza AM, et al. Mitochondrial DNA mutation associated with Leber's hereditary optic neuropathy. Science. 1988; 242(4884): 1427-1430.

27. King MP, Attardi G. Human cells lacking mtDNA: Repopulation with exogenous mitochondria by comple-mentation. Science. 1989; 246(4929): 500-503. 